Check for updates

Cite this: Phys. Chem. Chem. Phys., 2017, 19, 21933

Received 26th June 2017,

Accepted 31st July 2017

DOI: $10.1039 / c 7 c p 04296 e$

rsc.li/pccp

\section{Polarization transfer efficiency in PHIP experiments $\dagger$}

\author{
M. Emondts, (D) ${ }^{a}$ J. F. P. Colell, (DD ${ }^{b}$ B. Blümich (D) ${ }^{a}$ and P. P. M. Schleker (D) acd $^{a}$
}

Parahydrogen induced polarization (PHIP) is a hyperpolarization method for NMR signal enhancement with applications in spectroscopy and imaging. Although parahydrogen can be easily enriched up to nearly $95 \%$, the polarization detected on the hydrogenated substrate is substantially lower, where numerous loss mechanisms between the start of the hydrogenation reaction and detection affect polarization levels. The quality of PHIP systems is commonly determined by stating either the polarization degree or the enhancement factor of the product at the time of detection. In this study, we present a method that allows the distinction of polarization loss due to both the catalytic cycle and $T_{1}$ relaxation of the formed product prior to detection. We determine the influence of homogeneous catalysts and define a rigorous measure of the polarization transfer efficiency (PTE). Our results show that the PTE strongly depends on the concentration of all components and the chemical structure of the catalyst as well as on the magnetic field of detection.

\section{Introduction}

The biggest challenge in Nuclear Magnetic Resonance (NMR) originates from low sensitivity as a result of low thermal spin polarization, which can be overcome by hyperpolarization methods. Dynamic Nuclear Polarization (DNP) ${ }^{1,2}$ and Parahydrogen Induced Polarization (PHIP), ${ }^{3-7}$ which can be hydrogenative (ALTADENA, PASADENA) ${ }^{5-8}$ or non-hydrogenative (Signal Amplification By Reversible Exchange, $\mathrm{SABRE}^{3,4}$ ), increase the NMR signal by many orders of magnitude.

In a hydrogenative PHIP experiment the spin order of parahydrogen $\left(p-\mathrm{H}_{2}\right)$, which can easily be enriched to nearly

\footnotetext{
${ }^{a}$ RWTH Aachen University, Institut für Technische und Makromolekulare Chemie, Worringer Weg 1, 52074 Aachen, Germany. E-mail: philipp.schleker@cec.mpg.de

${ }^{b}$ Duke University, Department of Chemistry, Durham, North Carolina 27708, USA

${ }^{c}$ Max-Planck-Institut für Chemische Energiekonversion, Stiftstr. 34-36,

45470 Mülheim an der Ruhr, Germany

${ }^{d}$ Institut für Energie und Klimaforschung (IEK-9), Forschungszentrum Jülich GmbH, 52425 Jülich, Germany

$\dagger$ Electronic supplementary information (ESI) available. See DOI: 10.1039/c7cp04296e
}

$95 \%$, corresponding to a hydrogen polarization of $>0.9$, is transferred to spins in a target molecule by a hydrogenation reaction in the presence of a catalyst. PHIP has become a valuable tool for the analysis of hydrogenation kinetics and reaction mechanisms ${ }^{9,10}$ and the application for molecular imaging $^{11-13}$ is emerging.

Despite the high polarization level of the parahydrogen the polarization of the hydrogenated substrate is commonly substantially lower. ${ }^{14,15}$ The net PHIP efficiency, or absolute polarization of the product compared to the initial $\mathrm{H}_{2}$ polarization, is limited by the kinetics of the catalytic processes as well as by the experimental NMR settings. In order to optimize the polarization retained on a target molecule, the sources and mechanisms of polarization loss have to be identified and quantified.

Let us consider a hydrogenation reaction with para enriched hydrogen in the presence of a homogeneous catalyst and in the absence of paramagnetic impurities (e.g. oxygen). Free parahydrogen is protected from polarization loss due to symmetry. ${ }^{16}$ In a catalytic hydrogenation the first step is the formation of a dihydride (or dihydrogen) transition metal complex as exemplified in Fig. 1. ${ }^{17,18}$ As soon as the parahydrogen molecule is bound to a catalyst it is no longer protected by symmetry. From this point on polarization is lost by different mechanisms. ${ }^{16,19-21}$ The hydrogenation reaction proceeds involving several, often undetectable, intermediates in the catalytic cycle. ${ }^{22-25}$ The product is formed with the effective hydrogenation rate constant $k_{\text {hyd }}$. If hyperpolarized (hp) product $\mathbf{P}^{*}$ is formed, it is subject to $T_{1}$ relaxation (Fig. 1) during the delay between product formation and detection. Therefore, the amount of total polarization loss depends on the relaxation rates and lifetimes of catalyst-bound hydrogen and substrate species as well as on the $T_{1}$ relaxation of the hyperpolarized product before detection.

In the following we will differentiate between polarization loss occurring during the lifetime of catalytic intermediates (Fig. 1, red circles) and $T_{1}$ relaxation of $\mathbf{P}^{*}$ (Fig. 1, grey box). The fraction of the polarization that is transferred from $p-\mathrm{H}_{2}$ to the product, excluding $T_{1}$ relaxation of $\mathbf{P}^{*}$ (or polarization transfer to heteronuclei), quantifies the limit of polarization attainable 


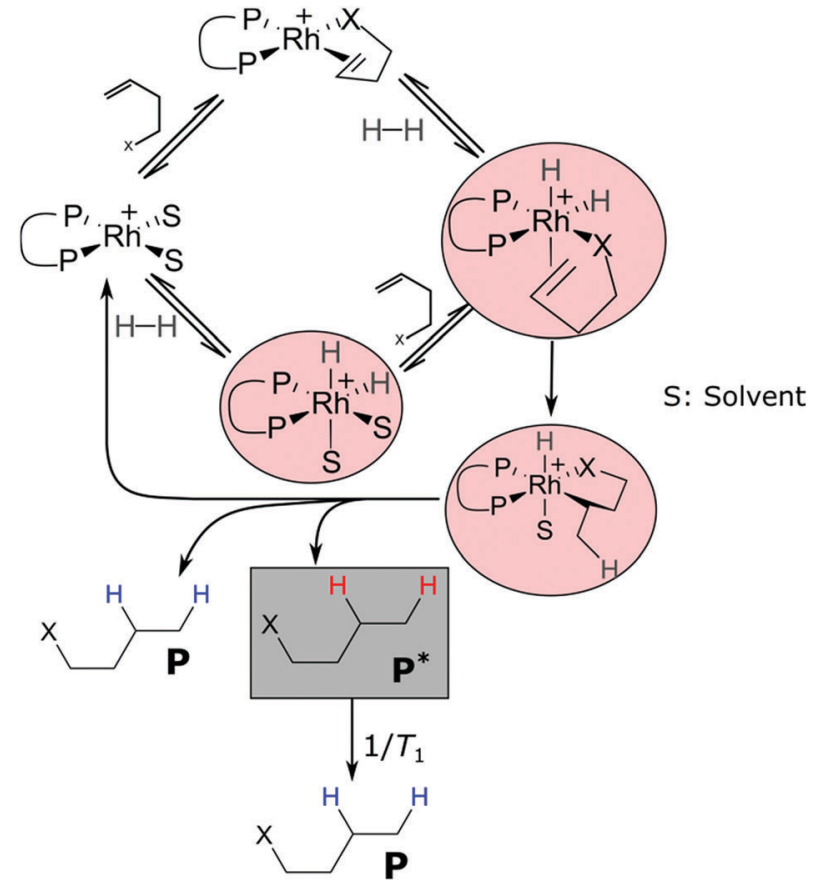

Fig. 1 Selected intermediates of the catalytic cycle, formation of either hp product $\mathbf{P}^{*}$ or tp product $\mathbf{P}$ and subsequent $T_{1}$ relaxation of $\mathbf{P}^{*}$ to $\mathbf{P}$. Polarization loss sources during the catalytic cycle are denoted in red, whereas the one after product formation is marked in grey.

on one particular type of nucleus in the ensemble of hydrogenated product molecules in a PHIP experiment and is thus a sharply defined measure of the polarization transfer efficiency (PTE). The shortcomings of the polarization degree and the enhancement factor are that neither the kinetics nor $T_{1}$ relaxation effects are considered, whereas the PTE offers a rigorous measure of the quality of PHIP systems. The advantage over the commonly used enhancement factor and polarization degrees at the time of detection is that polarization loss that can be attributed to the catalytic cycle and loss that originates from $T_{1}$ relaxation of $\mathbf{P}^{*}$ can be distinguished. This distinction allows optimization procedures for the chemical system as well as for the experimental procedure in order to obtain maximal polarization.

In the following we will introduce an easy method for the determination of the PTE. In the Experimental section we investigate the influence of the catalyst concentration and choice of catalysts as well as the effect of the magnetic field under PASADENA ${ }^{8}$ conditions on the PTE.

\section{Methods}

As introduced in the previous section, the PTE is the amount of polarization transferred from para enriched $\mathrm{H}_{2}$ to the product protons. We determine the PTE by comparing the experimental amount of substance of hyperpolarized product molecules $\mathbf{P}^{*}$ $\left(n_{\mathrm{P}^{*}, \exp }\right)$ at the time of detection to the idealized case without polarization loss $\mathbf{P}^{*}\left(n_{\mathrm{P}^{*}, i}\right)$, where $n_{\mathrm{P}^{*}, i}$ includes correction for longitudinal relaxation of $\mathbf{P}^{*}$ between product formation and detection (Fig. 1). Hence, the determination of $n_{\mathrm{P}^{*}, i}$ requires knowledge of the $T_{1}$ constant of $\mathbf{P}^{*}$ and of the chemical kinetics to determine the molar amount of hydrogenated substrates at the time of detection. If kinetic studies are performed at a high field under PASADENA ${ }^{8}$ conditions, the polarization transfer from the hp protons to naturally abundant ${ }^{13} \mathrm{C}$ is negligible, polarization transfer by isotropic mixing is inefficient ${ }^{26}$ and $n_{\mathrm{P}^{*}, \exp }$ is determined via line shape analysis of the PASADENA spectrum. The determination of the PTE is a four-step process:

(1) Determination of hydrogenation kinetics and $T_{1}$

(2) Determination of the number of $\mathbf{P}^{*}$ for the ideal case $\left(n_{\mathrm{P}^{*}, i}\right)$ where polarization loss occurs solely due to $T_{1}$ relaxation of $\mathbf{P}^{*}$ (Fig. 1)

(3) Determination of the molar amount of the experimentally observed $\mathbf{P}^{*}\left(n_{\mathbf{P}^{*}, \exp }\right)$

(4) Determination of the PTE by comparing $n_{\mathrm{P}^{*}, \exp }$ (step 3) to $n_{\mathrm{P}^{*}, i}($ step 2)

\section{Step 1}

If the chemical reaction is carried out with a large excess of substrate the kinetics are described by a first order process. By working in a closed vessel the hydrogen amount is limited. The kinetics of the hydrogenation reaction is recorded by applying consecutive 45 degree pulses separated by a time delay $\Delta t$ (Fig. 2a). ${ }^{9}$ The time delay $\Delta t$ is chosen large enough to allow the spins to realign before the next pulse application. The total molar amount of product $n_{\text {tot }}$ is the sum of hp molecules $\mathbf{P}^{*}$ and thermally polarized (tp) molecules $\mathbf{P}\left(n_{\mathrm{tot}}(t)=n_{\mathrm{P}^{*}}(t)+n_{\mathrm{P}}(t)\right)$. For a first order process we have:

$$
n_{\mathrm{tot}}(t)=n_{\infty}\left(1-\mathrm{e}^{-k_{\mathrm{hyd}} t}\right),
$$

here, $n_{\infty}$ denotes the overall yield at the end of the hydrogenation reaction and $k_{\text {hyd }}$ represents the rate constant of the hydrogenation. Eqn (1) enables the calculation of the amount of molecules hydrogenated in each time interval $\Delta t$ :

$$
\Delta n(t)=n_{\text {tot }}(t)-n_{\text {tot }}(t-\Delta t) .
$$

The $T_{1}$ constant of the product protons is accessible by an inversion recovery experiment.

\section{Step 2}

Let us consider an ideal case, where no polarization is lost during the catalytic cycle $(\mathrm{PTE}=1)$ but only due to $T_{1}$ relaxation between hydrogenation and acquisition. a)

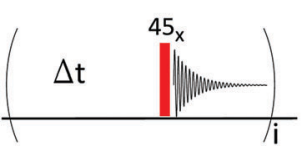

b)

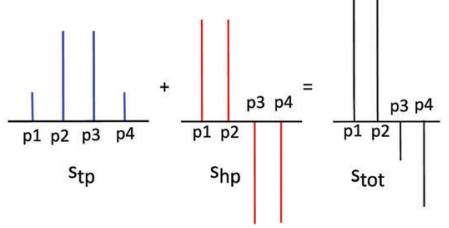

Fig. 2 Experimental procedure and processing. (a) Pulse sequence. (b) Line shape analysis. 
The number of $\mathbf{P}^{*}$ at the time of detection will be defined by the initial fraction of hp hydrogen and the reaction rate $k_{\text {hyd }}$ (as polarization supply rate) as well as by the relaxation rate $T_{1}$ (as a polarization loss mechanism). The initial fraction $x_{\mathrm{hp}}^{0}$ of hp hydrogen can be calculated with the para enrichment factor $x_{\text {para }}$ (see the ESI $\dagger$ for the derivation).

$$
x_{\mathrm{hp}}^{0}=x_{\text {para }}-\frac{1-x_{\text {para }}}{3} .
$$

For $x_{\text {para }}=0.93$ the hp fraction is $x_{\mathrm{hp}}^{0}=0.9$. This means that $10 \%$ of the amount of hydrogen is tp (representing the fraction of hydrogen containing $25 \%$ para and $75 \%$ ortho) and $90 \%$ is hp (displaying the remaining excess of para hydrogen). For $\mathrm{PTE}=1$, the molar amount of $\mathbf{P}^{*}$ formed during each time interval is determined by $\Delta n(t) x_{\mathrm{hp}}^{0}$. As soon as $\mathbf{P}^{*}$ is formed, it is subject to $T_{1}$ relaxation (Fig. 1 ). If we account for $T_{1}$ relaxation and the chemical kinetics, the molar amount $n_{\mathbf{P}^{*}, i}(t)$ of $\mathbf{P}^{*}$ at the time of each pulse can be described by:

$$
n_{\mathrm{P}^{*}, i}(t)=\frac{k_{\mathrm{hyd}} \Delta n(t) x_{\mathrm{hp}}^{0}}{1 / T_{1}-k_{\mathrm{hyd}}}\left(\mathrm{e}^{-k_{\mathrm{hyd}} \Delta t}-\mathrm{e}^{-\Delta t / T_{1}}\right) .
$$

Eqn (4) is equivalent to the kinetics of a first order consecutive reaction; ${ }^{9,27}$ the hydrogenation reaction with the effective rate constant $k_{\text {hyd }}$ is followed by $T_{1}$ relaxation with the rate constant $1 / T_{1}$ (Fig. 1). $n_{\mathrm{P}^{*}, i}$ can be calculated, when $k_{\text {hyd }}, T_{1}$ and $\Delta t$ are known.

\section{Step 3}

The determination of the experimentally observed amount of hyperpolarized product molecules $n_{\mathrm{P}^{*} \text {,exp }}$ includes the analysis of the NMR signal. The NMR signal intensity $s_{\text {tp }}$ originating from tp molecules is given by their spin density (proportional to the amount of substances $n_{\mathrm{tp}}$ ) and the magnetic field strength defining their thermal polarization $P_{\mathrm{tp}}\left(s_{\mathrm{tp}} \propto n_{\mathrm{P}}(t) P_{\mathrm{tp}}\right)$. The same applies for the hyperpolarized species $\left(s_{\mathrm{hp}} \propto n_{\mathrm{P}^{*}}(t) P_{\mathrm{hp}}\right)$. The ratio of the signal intensities $s_{\mathrm{hp}} / s_{\mathrm{tp}}$ is equal to their respective signal fractions $x_{\mathrm{s}, \mathrm{hp}} / x_{\mathrm{s}, \mathrm{tp}}$ and is given by:

$$
\frac{s_{\mathrm{hp}}}{s_{\mathrm{tp}}}=\frac{n_{\mathrm{P} *} P_{\mathrm{hp}}}{n_{\mathrm{P}} P_{\mathrm{tp}}}=\frac{x_{\mathrm{s}, \mathrm{hp}}}{x_{\mathrm{s}, \mathrm{tp}}} .
$$

Under PASADENA conditions the maximal achievable hyperpolarization degree $P_{\mathrm{hp}}$ equals 0.5 as the population of the initial singlet state is equally distributed between the $|\alpha \beta\rangle$ and $|\beta \alpha\rangle$ states, leading to a maximal polarization of 0.5 instead of 1 for the ALTADENA case.

In order to determine the individual fractions of hp and tp signals $\left(x_{\mathrm{s}, \mathrm{hp}}\right.$ and $\left.x_{\mathrm{s}, \mathrm{tp}}\right)$, the quartet of the product peak arising from the methylene-group is analyzed (Fig. 2b). The relative peak intensities $I$ are $1: 3: 3: 1$ for the thermally polarized case and $1: 1:-1:-1$ for the hyperpolarized one. As exemplified in Fig. 2b, the total NMR signal is a superposition of thermal and hyperpolarized signals. The ratio $r_{12}=r_{34}$ of the peak intensities in the quartet allows the calculation of the fraction of hyperpolarized signal $x_{\mathrm{s}, \mathrm{hp}}$ and thermally polarized signal $x_{\mathrm{s}, \mathrm{tp}}$ :

$$
r_{12}=\frac{I(\mathrm{p} 1)}{I(\mathrm{p} 2)}=\frac{1}{3} x_{\mathrm{s}, \mathrm{tp}}+x_{\mathrm{s}, \mathrm{hp}} .
$$

With $x_{\mathrm{s}, \mathrm{tp}}+x_{\mathrm{s}, \mathrm{hp}}=1$, the fraction of the hp signal is $x_{\mathrm{s}, \mathrm{hp}}=\left(3 r_{12}-1\right) / 2$ and of the tp signal is $x_{\mathrm{s}, \mathrm{tp}}=\left(3 r_{12}-3\right) / 2$. The number of experimentally observed $\mathbf{P}^{*}$ molecules $\left(n_{\mathbf{P}^{*}, \exp }\right)$ at the time of each pulse application can be determined by rearranging eqn (5) and merging it to $n_{\text {tot }}(t)=n_{\mathrm{P}^{*}}(t)+n_{\mathrm{P}}(t)$ :

$$
n_{\mathrm{P}^{*}, \exp }(t)=\frac{n_{\mathrm{tot}}(t)}{\left(1+\frac{x_{\mathrm{s}, \mathrm{hp}} P_{\mathrm{tp}}}{x_{\mathrm{s}, \mathrm{tp}} P_{\mathrm{hp}}}\right)} .
$$

\section{Step 4}

The polarization transfer efficiency (PTE) is simply the ratio of $n_{\mathrm{P}^{*}, \exp }$ (eqn (7)) to $n_{\mathrm{P}^{*}, i}$ (eqn (4)),

$$
\mathrm{PTE}=\frac{n_{\mathrm{P}^{*}, \exp }(t)}{n_{\mathrm{P}^{*}, i}(t)},
$$

and defines the limit of polarization attainable on one particular type of nucleus in the ensemble of hydrogenated product molecules in a PHIP experiment quantitatively.

Note that the determination of the PTE requires only the determination of the effective hydrogenation rate $k_{\text {hyd }}$ and one $T_{1}$ measurement. The time dependent increase of a tp product peak (or the decrease of the educt peak) allows the determination of the reaction constant $k_{\text {hyd }}$. Once $T_{1}$ and $k_{\text {hyd }}$ are known, $n_{\mathrm{P}^{*}, i}$ can be calculated according to eqn (4). This number is then compared to the experimentally observed value $n_{\mathrm{P}^{*} \text {,exp }}$, which can be determined by line shape analysis of the antiphase quartet.

\section{Experimental}

In this study the precatalysts [Rh(COD)BINAP]BF 4 (BINAP: $\left(2,2^{\prime}\right.$ bis(diphenylphosphino)-1,1'-binaphthyl), COD: cyclooctadiene) and [Rh(COD)DPPB $] \mathrm{BF}_{4}$ (DPPB: (1,4-bis(diphenylphosphino)butane)) were used to investigate the PTE. The complexes were synthesized using published procedures. ${ }^{28}$ The solvent was $\mathrm{d}_{4}-\mathrm{MeOH}$, the substrate butyl acrylate $(c=0.5 \mathrm{M})$. All samples were prepared in Wilmad medium pressure NMR tubes under inert conditions. The NMR tube was pressurized with 2 bar parahydrogen. After pressurizing, the samples were shaken for five seconds and transferred to the magnetic detection field (Bruker AV III 300, 7.05 T, or Bruker AV II 600, $14.09 \mathrm{~T}$, spectrometer). The kinetics of the hydrogenation reaction (Step 1) was recorded by applying consecutive $45^{\circ}$ pulses in time intervals $\Delta t$ (Fig. 2a). All experiments were conducted at $25{ }^{\circ} \mathrm{C}$.

\section{Results}

In the experimental study, we investigated the influence of the catalyst concentration, the magnetic field and the chemical structure of the catalyst on the PTE. For each experiment the PTE was determined for the time course of the hydrogenation 
a)

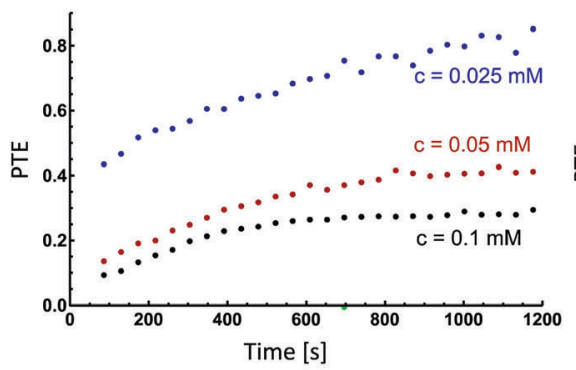

b)

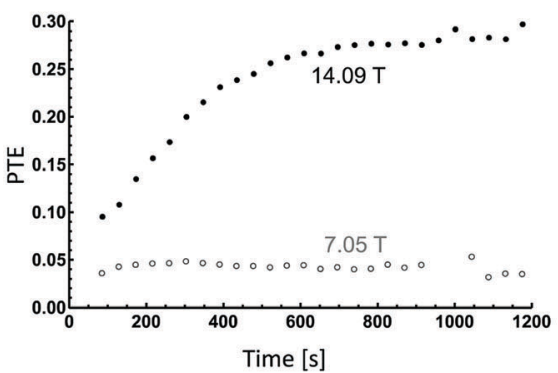

c)

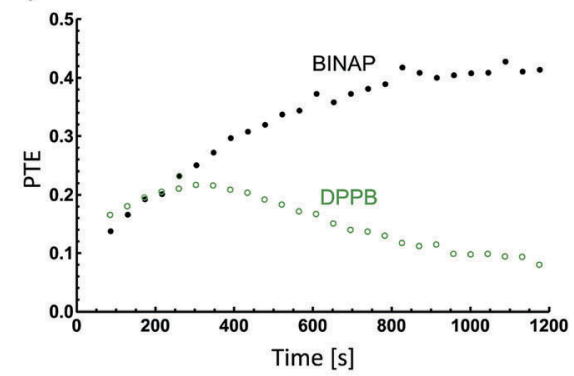

Fig. 3 Time course of the PTE for (a) different catalyst concentrations of $[R h(C O D) B I N A P] B F_{4}$ at $14.09 \mathrm{~T}$, (b) $[R h(C O D) B I N A P] B F_{4}\left(c=0.1 m_{M}\right)$ at $7.05 T$ and 14.09 T detection field, (c) for [Rh(COD)BINAP]BF 4 and [Rh(COD)DPPB]BF 4 at $14.09 \mathrm{~T}$.

as described in the Methods section. The kinetic parameters as well as the detailed processing procedure are presented in the ESI. $\dagger$

We recorded the kinetics of hydrogenation reactions at different concentrations of [Rh(COD)BINAP $] \mathrm{BF}_{4}$ and determined the PTE as a function of time (Fig. 3a). The initial efficiency is the highest for the lowest catalyst concentration with about $40 \%$ at the beginning, while the other concentrations gain less than $18 \%$ at this point. This series shows a tendency of increasing efficiency with decreasing catalyst concentration. Furthermore, the PTE rises with time for all solutions, which can be connected to dropping $\mathrm{H}_{2}$ pressure. Depending on the concentration of the alkene and $\mathrm{H}_{2}$, the former or latter is coordinated longer to the catalytic centre. ${ }^{17,29}$ The coordination of $p-\mathrm{H}_{2}$ as classical or non-classical hydride opens up the possibility for a relaxation pathway. The dihydrides, in particular the non-classical hydrides, have short $T_{1}$ relaxation times. $T_{1}$ is on the order of milliseconds and tends to be longer in higher magnetic fields. ${ }^{23}$

Hence, changing the magnetic field of detection allows gaining more insight into polarization loss mechanisms. In order to investigate the influence of $T_{1}$ relaxation of hydride intermediates on the PTE samples of identical composition were analysed in two magnetic fields (7.05 $\mathrm{T}$ and $14.09 \mathrm{~T}$ ). When performing the hydrogenation reaction at $14.09 \mathrm{~T}$ the PTE increases from 0.08 to nearly 0.3 as the reaction proceeds (Fig. 3b). At 7.05 T the PTE increases only slightly from 0.04 to 0.06 . This clearly shows that $T_{1}$ relaxation of the intermediates prior to chemical reaction constitutes a highly relevant loss pathway.

To elucidate upon the effect of the catalyst structure on the PTE, we changed the ligand from BINAP to DPPB. The concentration of DPPB was adjusted for the effective hydrogenation rates $k_{\text {hyd }}$ to match $\left(c\left([\mathrm{Rh}(\mathrm{COD}) \mathrm{BINAP}] \mathrm{BF}_{4}\right)=0.05 \mathrm{mM}\right.$, $\left.c\left([\mathrm{Rh}(\mathrm{COD}) \mathrm{DPPB}] \mathrm{BF}_{4}\right)=0.01 \mathrm{mM}\right)$. If we compare the systems the initial PTE is around 0.15 for both (Fig. 3c), showing that the ligand has a significant impact, as one would expect the lower concentrated and faster hydrogenating catalyst to have a higher PTE. Interestingly, the PTE of DPPB increases at first, but declines after approximately $350 \mathrm{~s}$, whereas the PTE of BINAP asymptotically increases from 0.12 to 0.4 . The decrease of the PTE over time is attributed to para-ortho ( $p / o$ ) hydrogen conversion due to its reversible coordination. ${ }^{16}$ This is also proven by higher $o-\mathrm{H}_{2} \mathrm{NMR}$ signal intensity with DPPB (see Fig. S5, ESI $\dagger$ ).

\section{Conclusions}

In this work we introduced the PTE as a parameter that quantifies the maximal fraction of polarization transferred from $p-\mathrm{H}_{2}$ to the product's protons regardless of subsequent $T_{1}$ relaxation of the hyperpolarized product prior to detection. We proposed a simple method based on kinetic investigations of the hydrogenation reaction that enables the quantification of the PTE in PHIP experiments. The experimental results show that the PTE is determined by the concentration of all components, the chemical structure of the catalyst as well as the magnetic field.

The PTE is a suitable parameter for the characterization of PHIP systems and can not only be used for the investigation of homogeneous, but also for heterogeneous systems. This characterization method is highly important for the fundamental understanding of the established PHIP systems and may also elucidate guidelines for the development of new efficient homogenous and heterogeneous PHIP catalysts and the transfer from batch to continuous flow. We believe this is particularly important in the development for biomedical applications. Future work will involve the investigation of catalysts that have been immobilized in order to analyse the influence of the supporting material on the PTE.

\section{Acknowledgements}

The authors would like to thank Jürgen Klankermayer and Ines Bachmann-Remy for support and technical assistance as well as Danila Barskiy for stimulating discussions. We thank S. Lehmkuhl for the provision of the catalyst. This work has been supported by Deutsche Forschungsgemeinschaft (DFG-BL231/47-1). Open Access funding provided by the Max Planck Society.

\section{References}

1 M. Abraham, M. A. H. McCausland and F. N. H. Robinson, Phys. Rev. Lett., 1959, 2, 449-451.

2 W. A. Barker, Rev. Mod. Phys., 1962, 34, 173-185. 
3 R. W. Adams, J. A. Aguilar, K. D. Atkinson, M. J. Cowley, P. I. P. Elliott, S. B. Duckett, G. G. R. Green, I. G. Khazal, J. Lopez-Serrano and D. C. Williamson, Science, 2009, 323, 1708-1711.

4 R. W. Adams, S. B. Duckett, R. A. Green, D. C. Williamson and G. G. R. Green, J. Chem. Phys., 2009, 131, 194505.

5 T. C. Eisenschmid, R. U. Kirss, P. P. Deutsch, S. I. Hommeltoft, R. Eisenberg, J. Bargon, R. G. Lawler and A. L. Balch, J. Am. Chem. Soc., 1987, 109, 8089-8091.

6 J. Natterer and J. Bargon, Prog. Nucl. Magn. Reson. Spectrosc., 1997, 31, 293-315.

7 C. R. Bowers and D. P. Weitekamp, Phys. Rev. Lett., 1986, 57, 2645-2648.

8 C. R. Bowers and D. P. Weitekamp, J. Am. Chem. Soc., 1987, 109, 5541-5542.

9 P. Hubler, R. Giernoth, G. Kummerle and J. Bargon, J. Am. Chem. Soc., 1999, 121, 5311-5318.

10 M. S. Chinn and R. Eisenberg, J. Am. Chem. Soc., 1992, 114, 1908-1909.

11 P. Nikolaou, B. M. Goodson and E. Y. Chekmenev, Chem. - Eur. J., 2015, 21, 3156-3166.

12 K. Golman, O. Axelsson, H. Jóhannesson, S. Månsson, C. Olofsson and J. S. Petersson, Magn. Reson. Med., 2001, 46, 1-5.

13 P. Bhattacharya, E. Y. Chekmenev, W. H. Perman, K. C. Harris, A. P. Lin, V. A. Norton, C. T. Tan, B. D. Ross and D. P. Weitekamp, J. Magn. Reson., 2007, 186, 150-155.

14 N. N. Jarenwattananon, S. Glöggler, T. Otto, A. Melkonian, W. Morris, S. R. Burt, O. M. Yaghi and L.-S. Bouchard, Nature, 2013, 502, 537-540.
15 R. V. Shchepin, A. M. Coffey, K. W. Waddell and E. Y. Chekmenev, J. Phys. Chem. Lett., 2012, 3, 3281-3285.

16 J. M. Brown, L. R. Canning, A. J. Downs and A. M. Forster, J. Organomet. Chem., 1983, 255, 103-111.

17 I. D. Gridnev and T. Imamoto, Acc. Chem. Res., 2004, 37, 633-644.

18 H. Heinrich, J. Bargon, R. Giernoth and J. M. Brown, Chem. Commun., 2001, 1296-1297.

19 G. Buntkowsky, B. Walaszek, A. Adamczyk, Y. Xu, H. H. Limbach and B. Chaudret, Phys. Chem. Chem. Phys., 2006, 8, 1929-1935.

20 J. Matthes, T. Pery, S. Gründemann, G. Buntkowsky, S. SaboEtienne, B. Chaudret and H.-H. Limbach, J. Am. Chem. Soc., 2004, 126, 8366-8367.

21 C. Terenzi, S. Bouguet-Bonnet and D. Canet, J. Phys. Chem. Lett., 2015, 6, 1611-1615.

22 I. D. Gridnev, N. Higashi, K. Asakura and T. Imamoto, J. Am. Chem. Soc., 2000, 122, 7183-7194.

23 P. G. Jessop and R. H. Morris, Coord. Chem. Rev., 1992, 121, 155-284.

24 J. Halpern, Inorg. Chim. Acta, 1981, 50, 11-19.

25 J. Halpern, Science, 1982, 217, 401-407.

26 S. Aime, R. Gobetto, F. Reineri and D. Canet, J. Chem. Phys., 2003, 119, 8890-8896.

27 C. T. Jones, Biochem. J., 1970, 118, 810-812.

28 A. Miyashita, A. Yasuda, H. Takaya, K. Toriumi, T. Ito, T. Souchi and R. Noyori, J. Am. Chem. Soc., 1980, 102, 7932-7934.

29 O. G. Salnikov, D. A. Barskiy, A. M. Coffey, K. V. Kovtunov, I. V. Koptyug and E. Y. Chekmenev, ChemPhysChem, 2016, 17, 3395-3398. 